\title{
Correction to: Influence of land use and artificial water bodies on the habitat use of Myocastor coypus and Hydrochoerus hydrochaeris in the Argentine Pampas
}

\author{
Maria Jose Corriale ${ }^{1,2}$ (D) Maria Eugenia Pedelacq ${ }^{1} \cdot$ María Laura Guichón $^{3} \cdot$ David Norberto Bilenca $^{1,2}$
}

Published online: 14 December 2020

(c) Deutsche Gesellschaft für Säugetierkunde 2020

\section{Correction to: Mammalian Biology \\ https://doi.org/10.1007/s42991-020-00082-2}

The original version of this article unfortunately contained a mistake. The following correction has therefore been made in the original: The spelling of David Norberto Bilenca's name was incorrect. The corrected author list is given above. The original article has been corrected.

The original article can be found online at https://doi.org/10.1007/ s42991-020-00082-2.

Maria Jose Corriale

mjcorriale@ege.fcen.uba.ar

1 Grupo de Estudios sobre Biodiversidad en Agroecosistemas, Departamento de Biodiversidad y Biología Experimental, Facultad de Ciencias Exactas y Naturales, Universidad de Buenos Aires, Ciudad Universitaria, Pabellón II, 4 Piso (C1428EHA), Ciudad Autónoma de Buenos Aires, Argentina

2 Instituto de Ecología Genética y Evolución de Buenos Aires (IEGEBA), UBA-CONICET,

Ciudad Autónoma de Buenos Aires, Argentina

3 Instituto de Investigaciones en Biodiversidad y Medioambiente (INIBIOMA, UNCo-CONICET), Sede Junín de los Andes, Centro de Ecología Aplicada del Neuquén (CEAN), Junín de los Andes, Neuquén, Argentina 